\title{
Thermal Runaway Studies for the CMS TFPX Phase-II Upgrade
}

\author{
$8 / 16 / 2021$ \\ Kevin Souhrada
}




\section{Outline:}

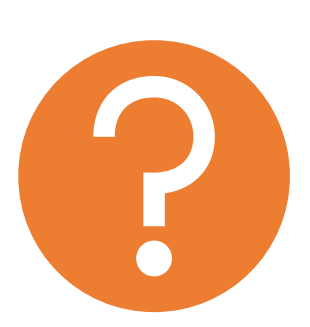

What is CMS/TFPX?

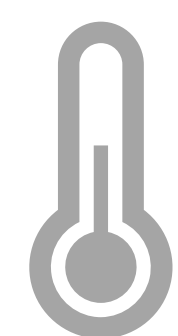

What is Thermal Runaway and how do we prevent it?
How do we simulate runaway in the lab? 
What is CMS/TFPX? 


\section{Compact Muon Solenoid (CMS)}

Muon

Detector

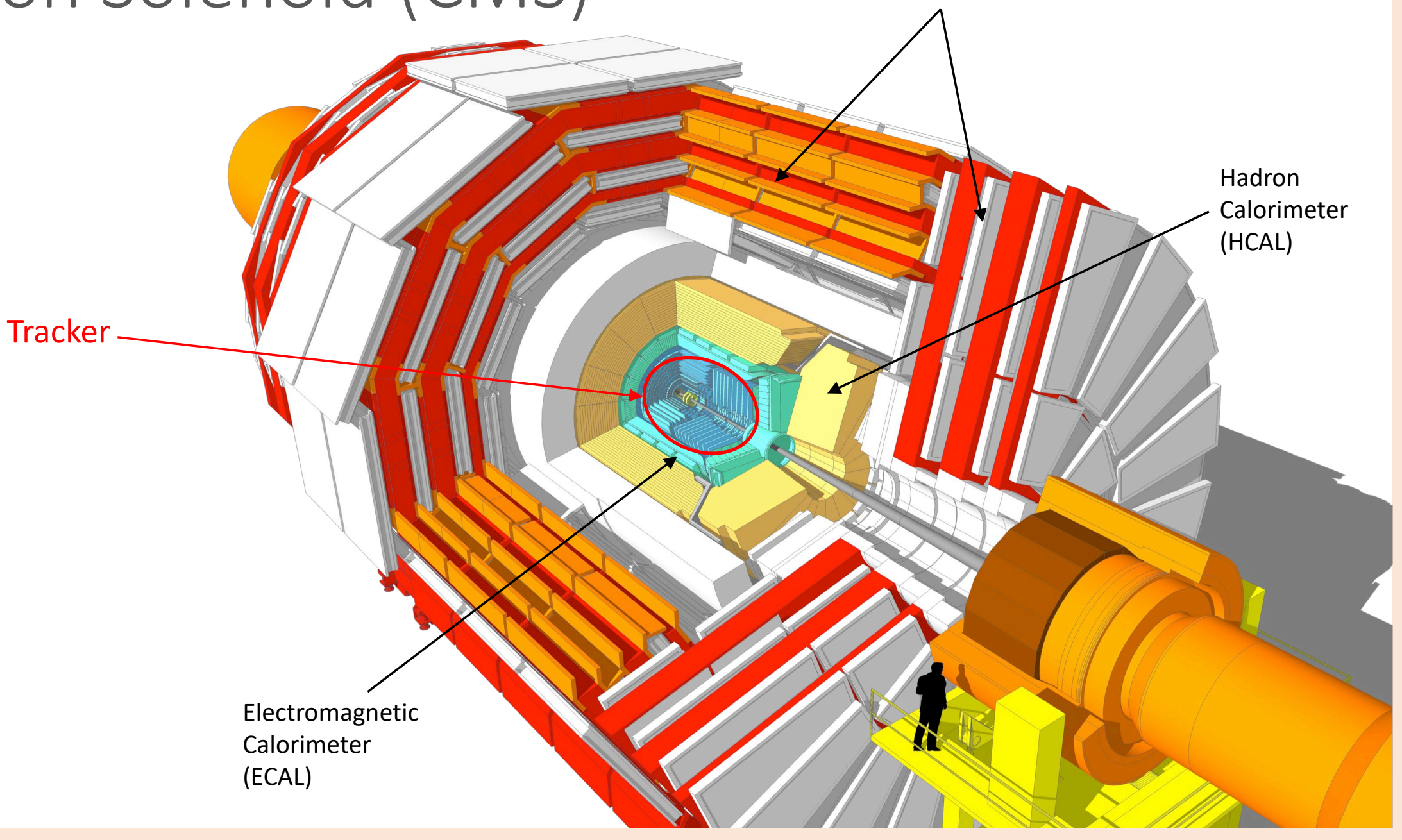




\section{CMS / Tracker}

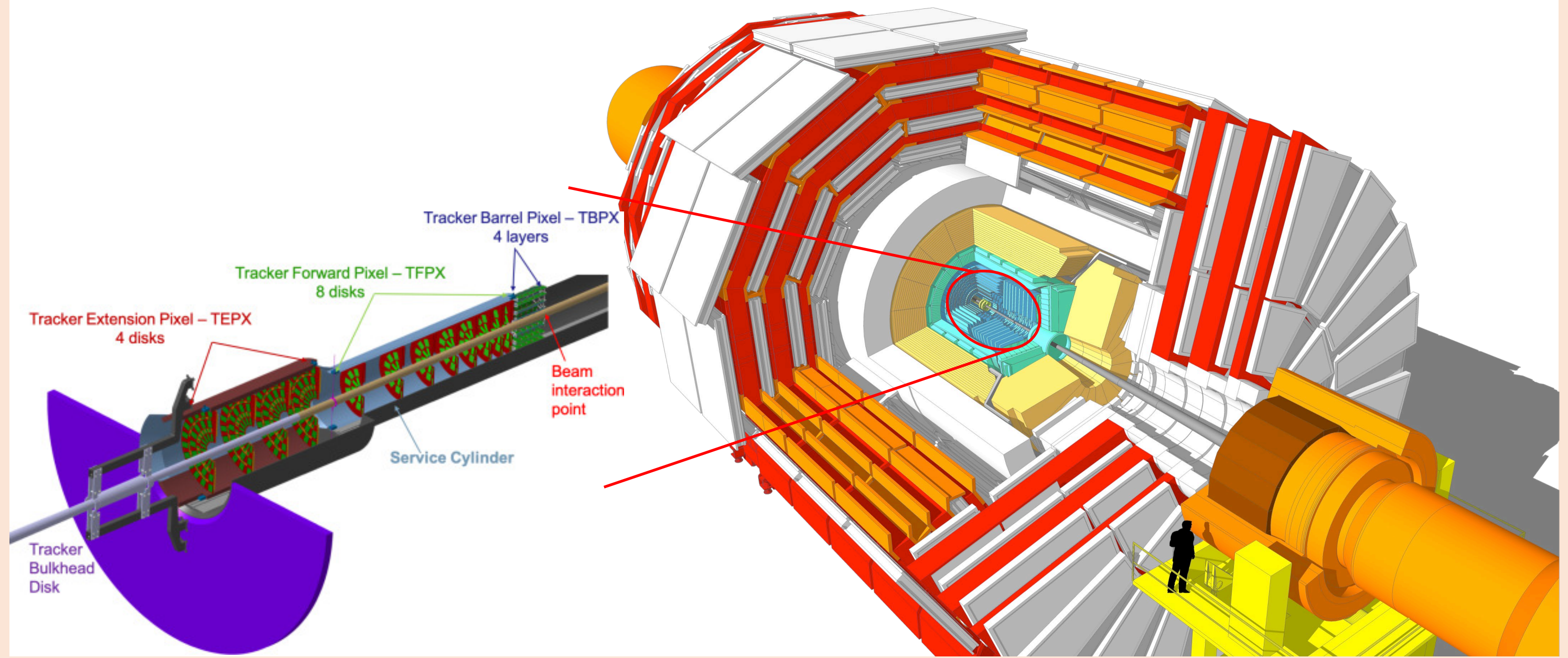




$$
\sigma
$$




\section{Tracker / TFPX}

\section{Innermost \\ subdetector}
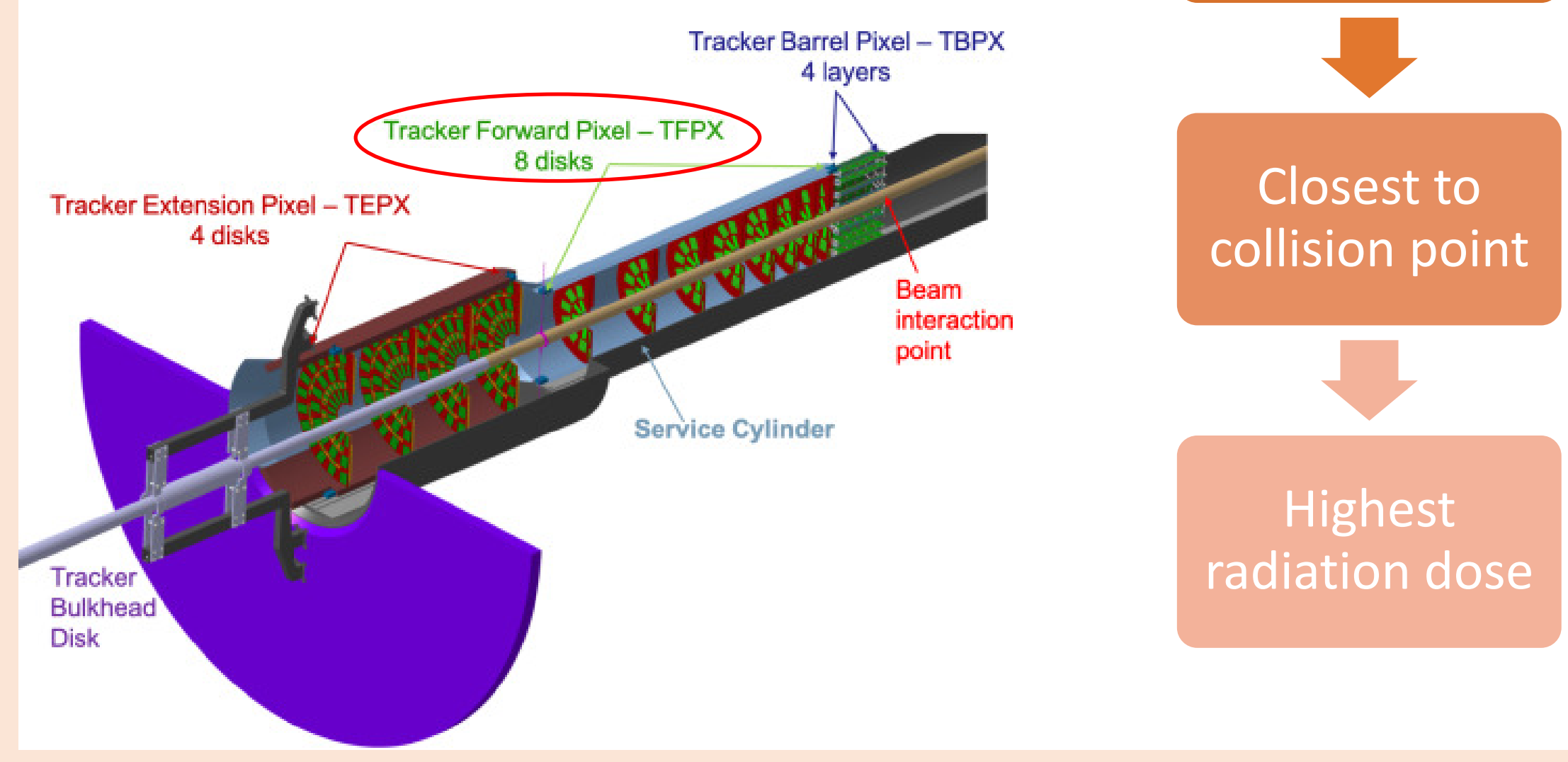

*Structure needs to be radiation hardy! 


\section{What is Thermal Runaway?}




\section{What is Thermal Runaway? (Chemistry)}

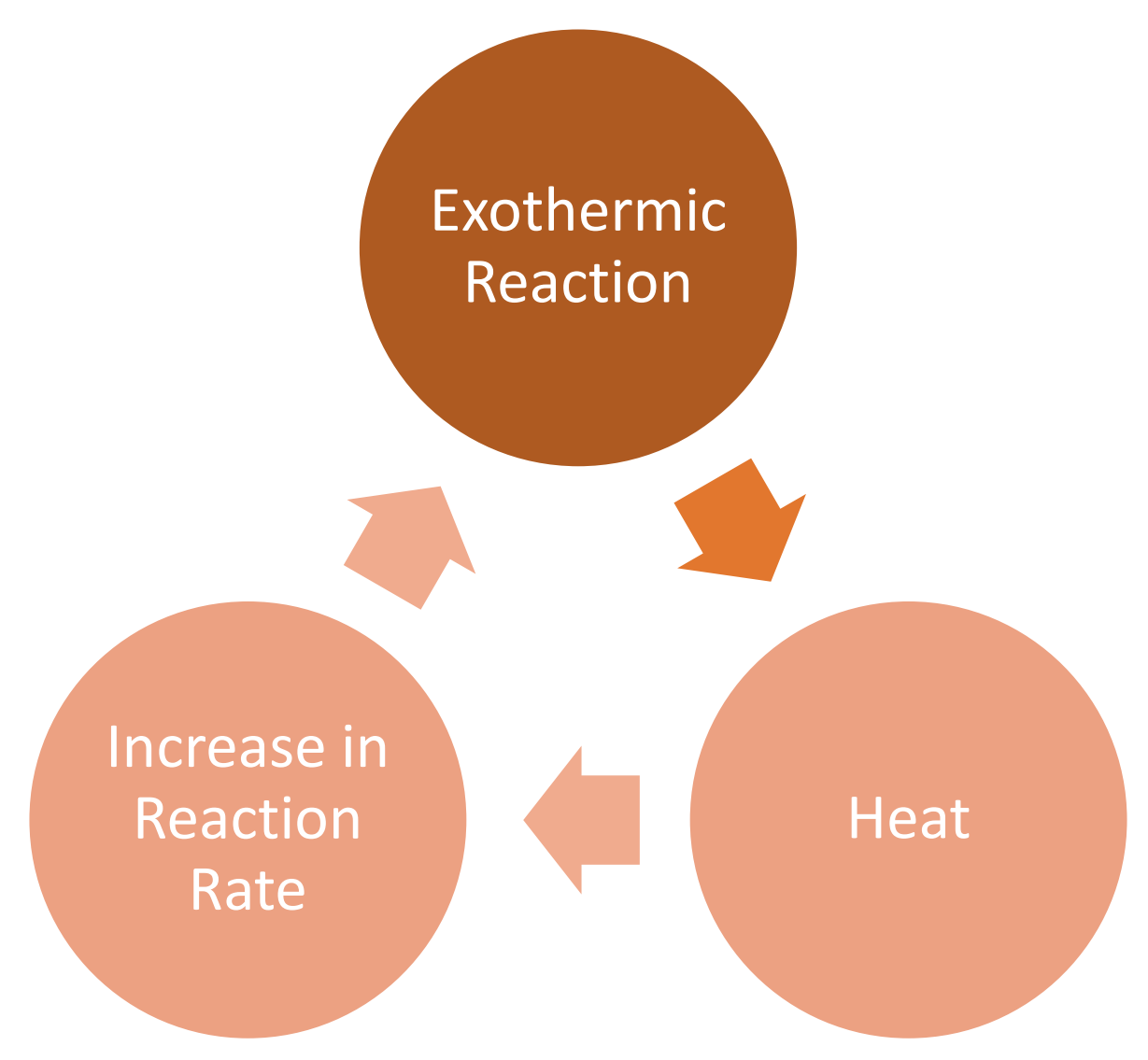




\section{What is Thermal Runaway? (TFPX)}

- Collisions happen very fast (every $25 n s$ )

- Huge amounts of data must be transferred at high speeds

- Electronics generate heat

- Dark current across the semiconductor of the silicon sensor

- With increased radiation damage, the silicon crystal is damaged allowing greater dark current to bridge the bandgap of the semiconductor

- Dark current generates ohmic heating, which in turn increases dark current, etc.

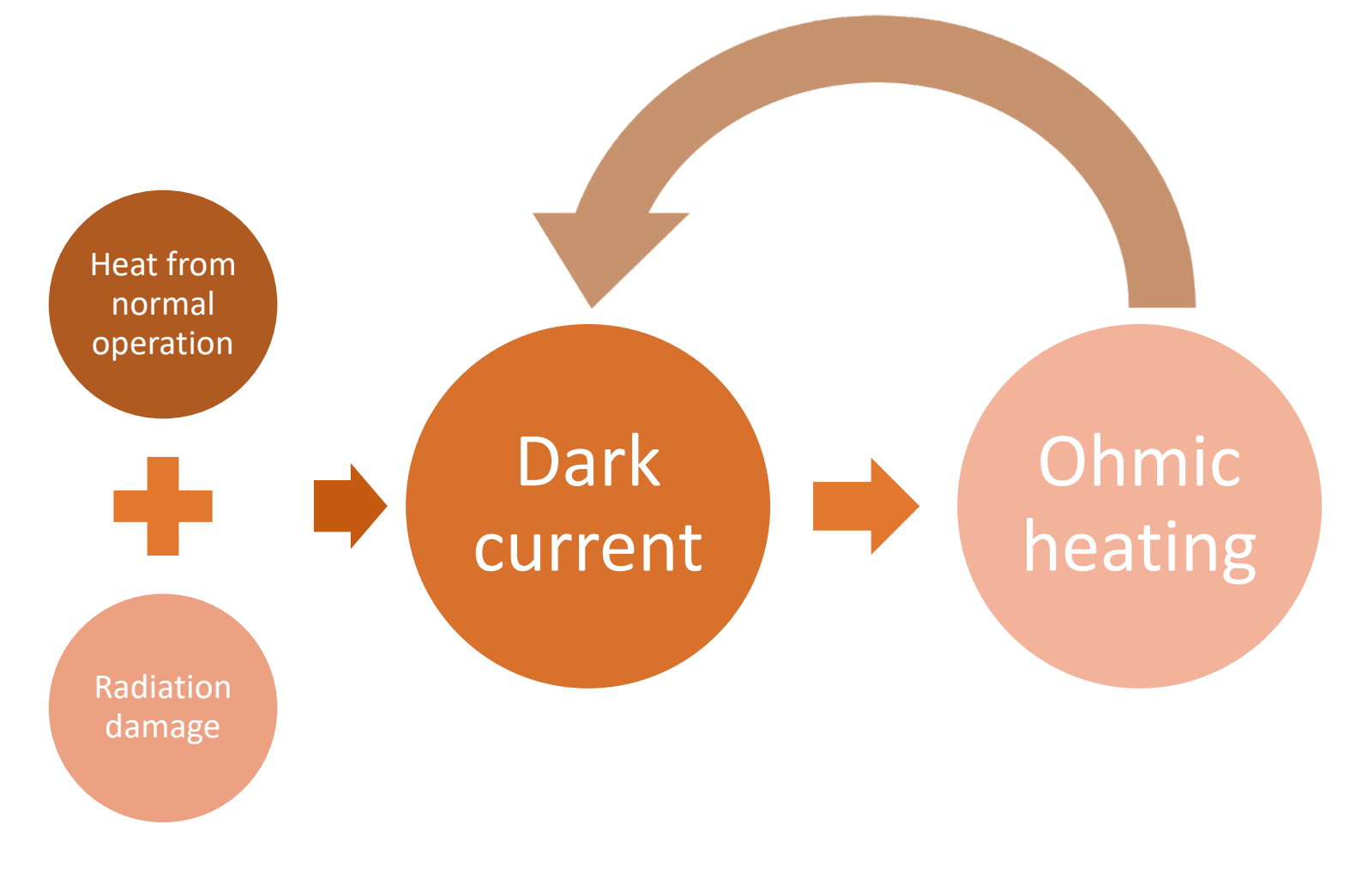


How do we prevent runaway? 


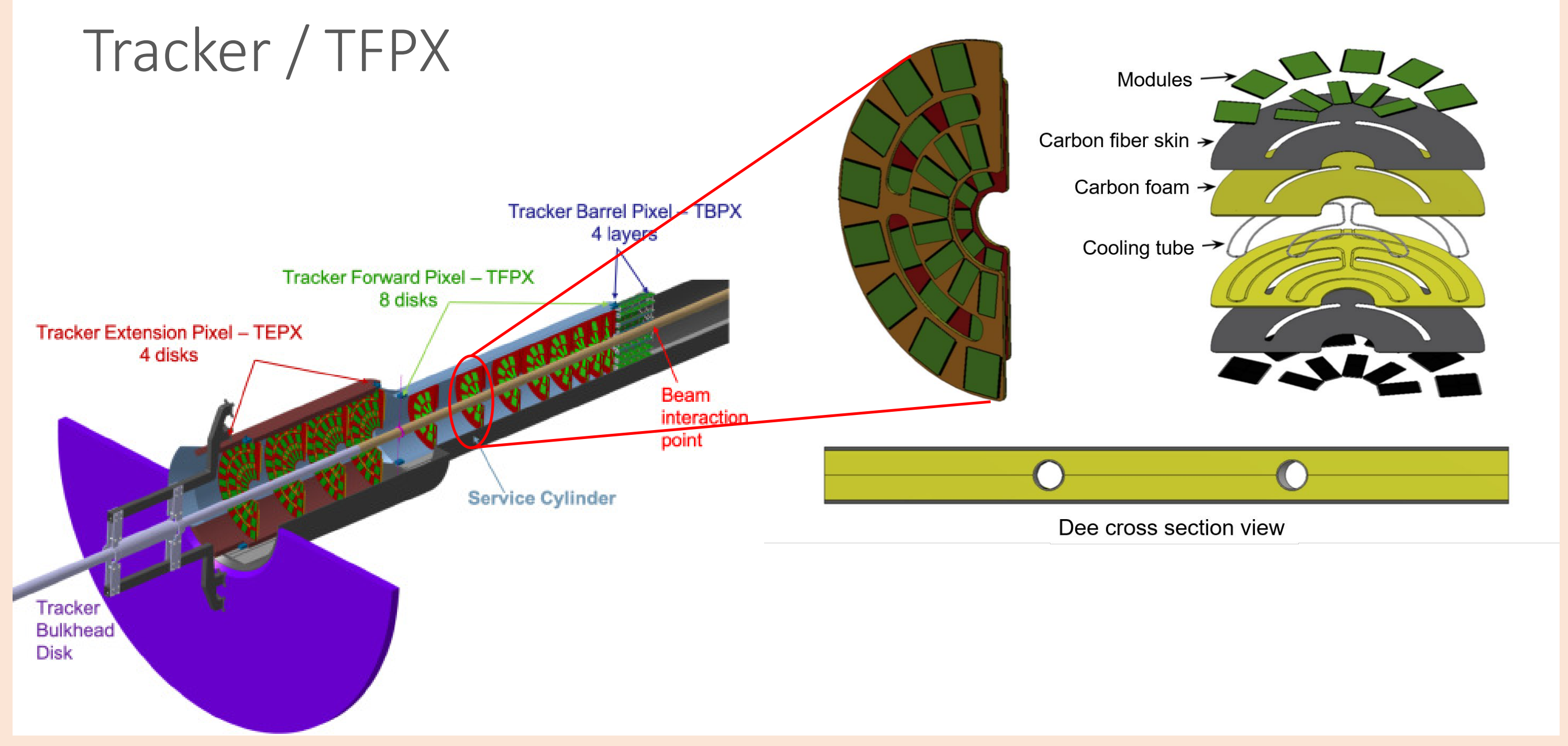




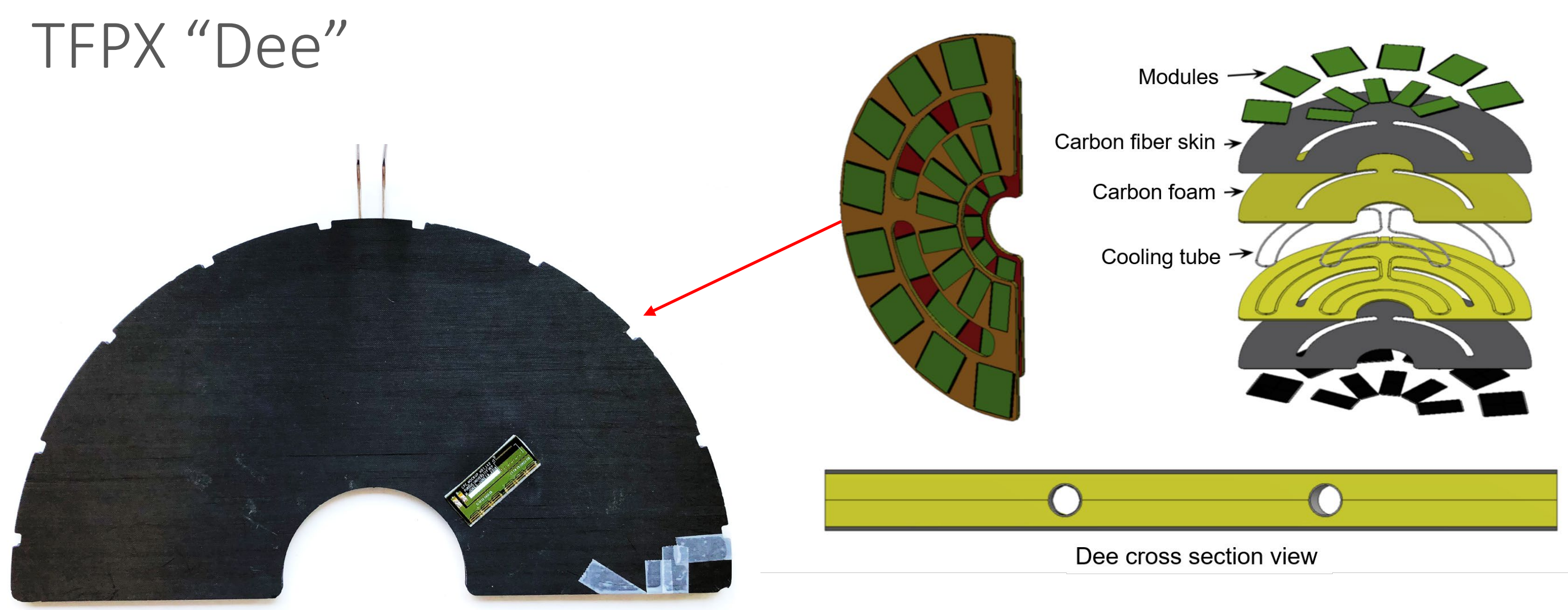




\section{How do we prevent Thermal Runaway?}

- Need to remove generated heat through the $\mathrm{CO}_{2}$ cooling

- The thermal impedance of the structure must be managed alongside several other design requirements (minimizing total mass, module interchangeability, radiation hardiness, etc.)

- One component of the structure that we can change:

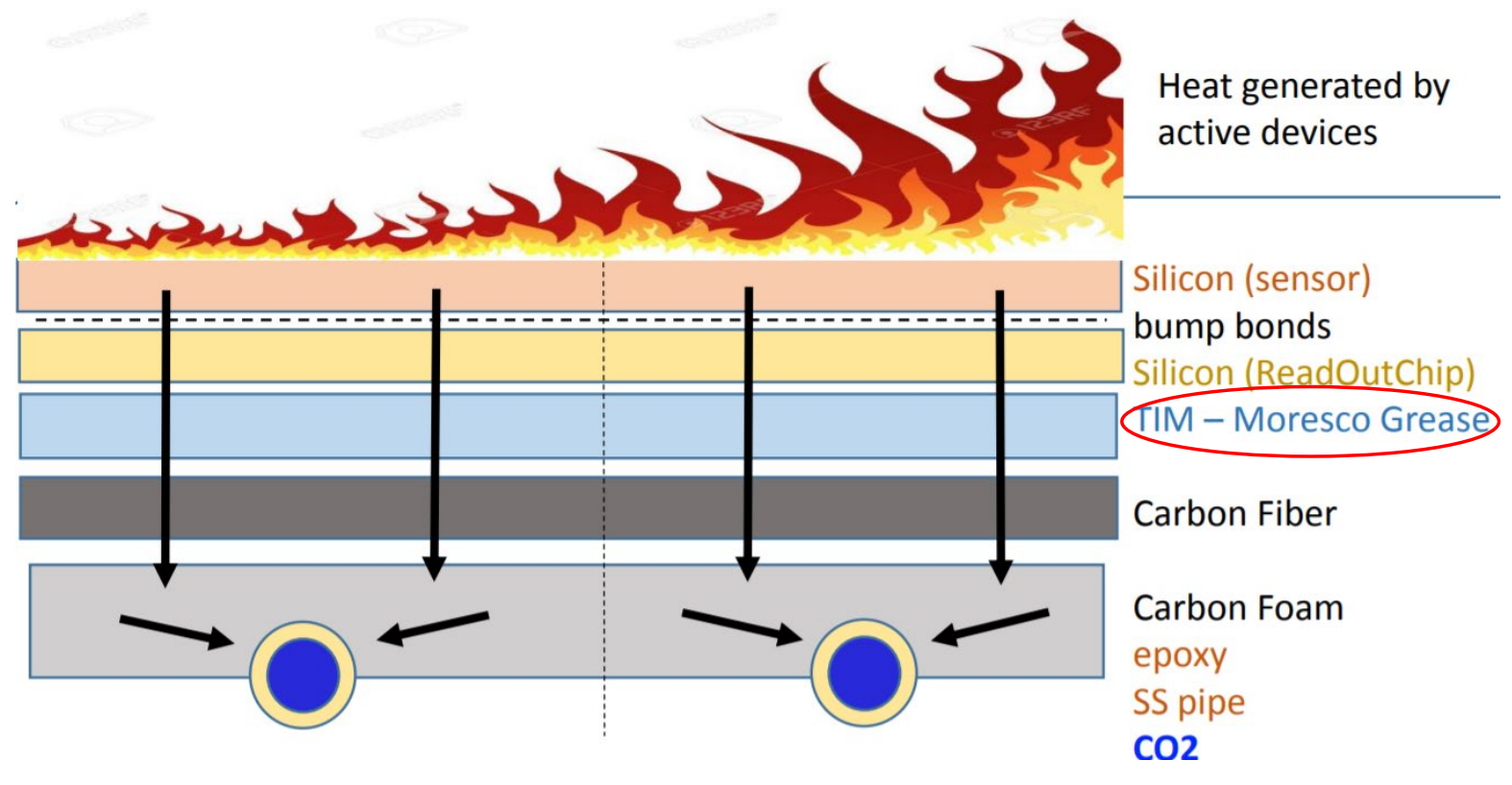

Thermal Interface Material (TIM) 
How do we simulate runaway? 


\section{How do we simulate Thermal Runaway?}
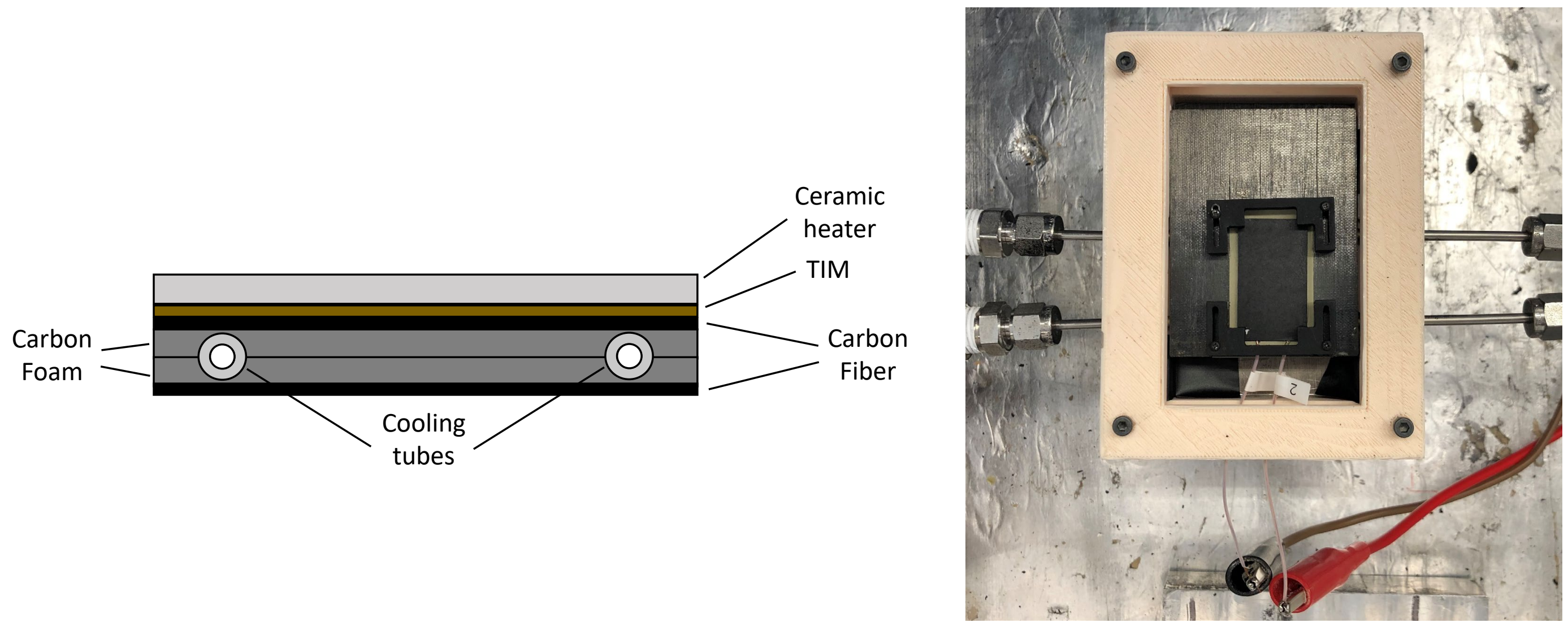


\section{How do we simulate Thermal Runaway?}

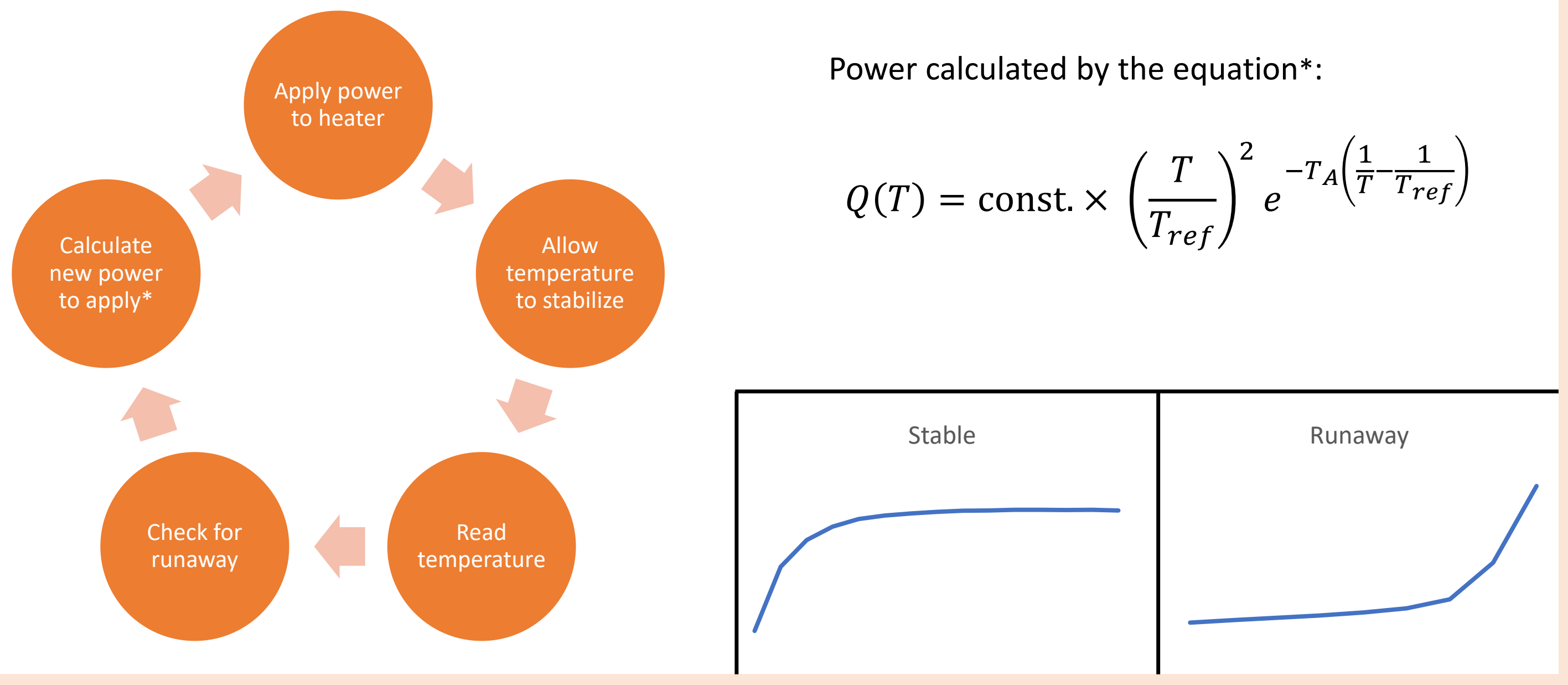




\section{Current runaway LabVIEW program}

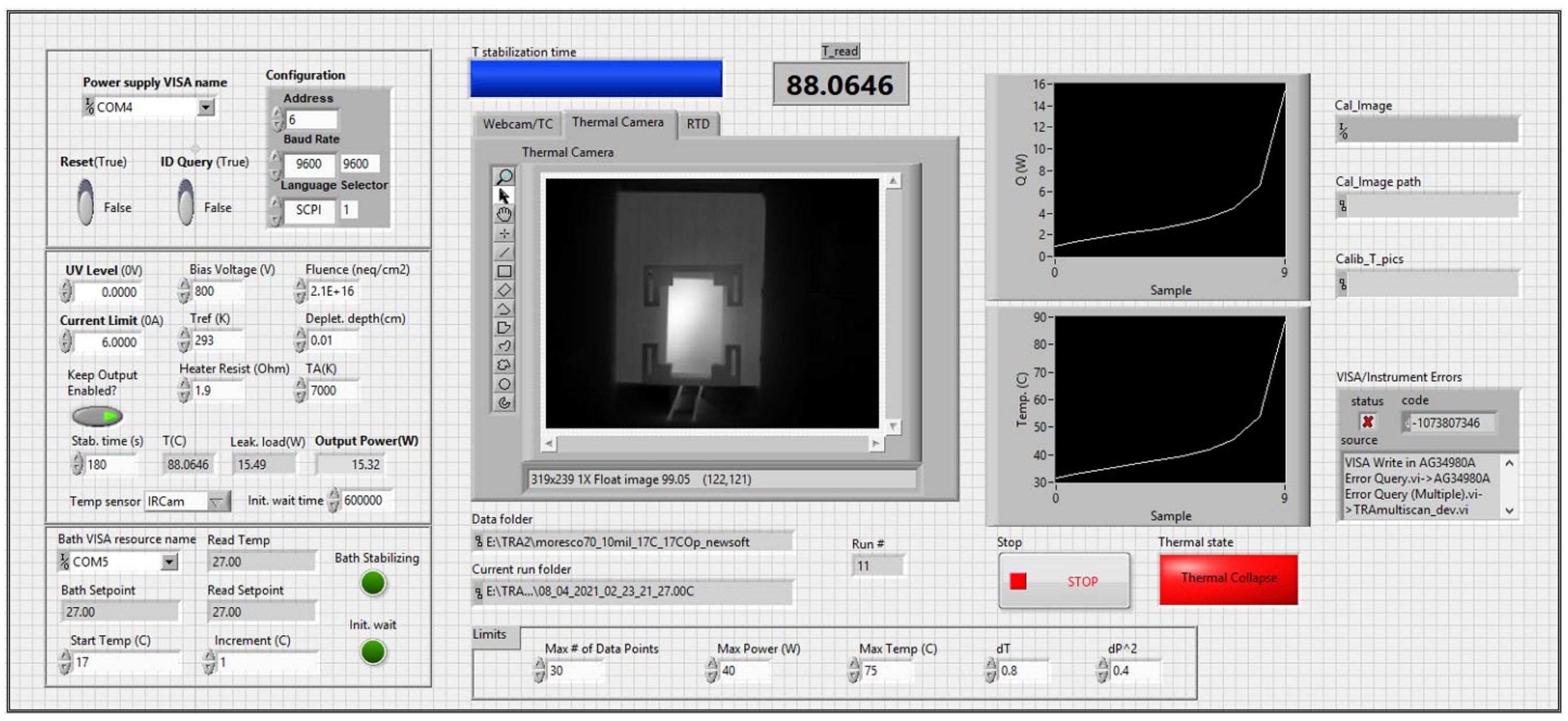




\section{Conclusions}

- Achieved a reproducible and systematic method for generating thermal runaway

- Simulating runaway allows us to determine the stable operating range for our final detector

- Our decisions on the final parameters of the mechanical structure of TFPX will be based on their thermal performance as determined through runaway testing

- Long term goals:

- Runaway testing on full-sized Dees with asymmetrically heated replica modules to fully model the detector environment 


\section{Thank you!}

Comments / questions? 


\section{Data}

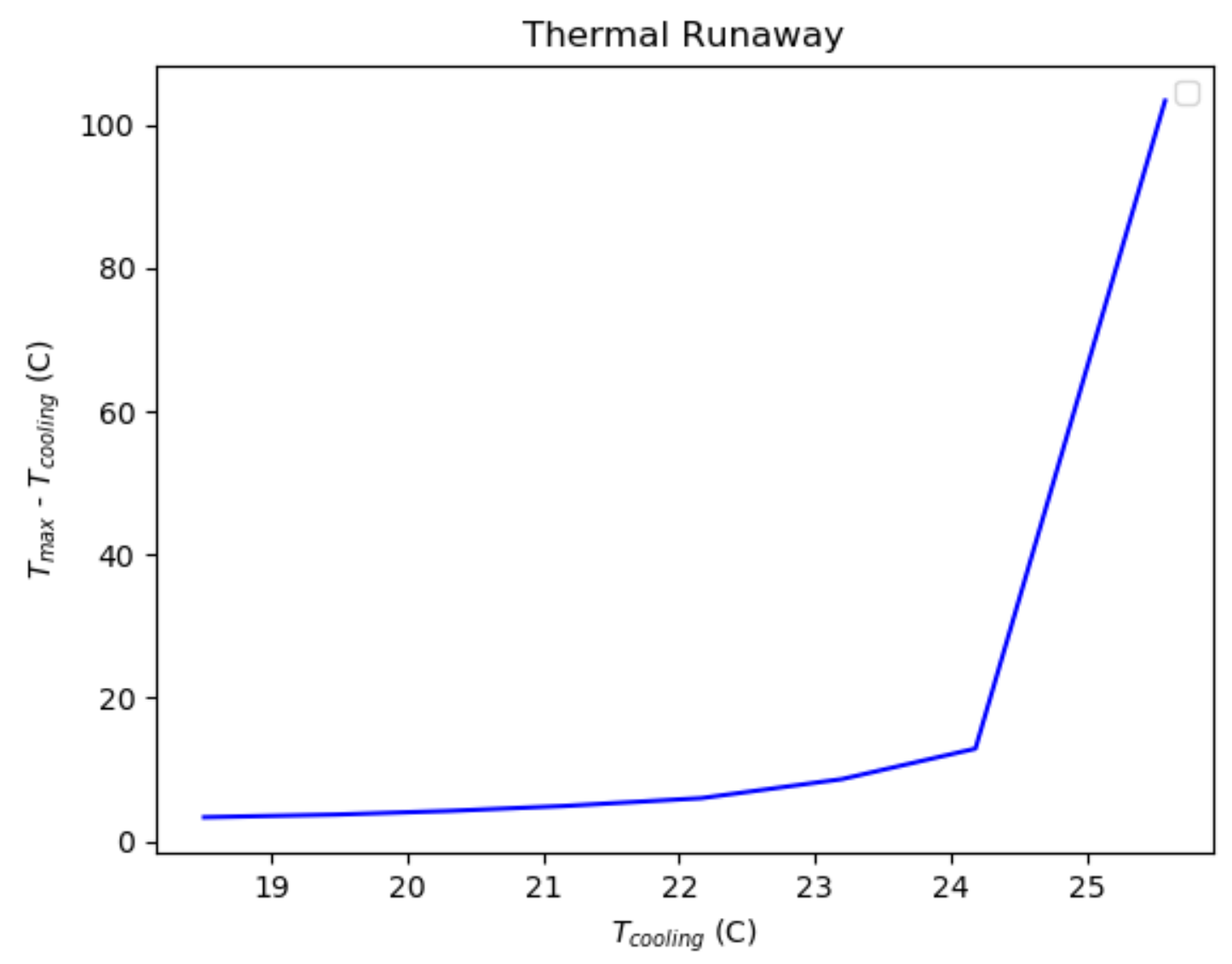

